\title{
Polarized light imaging specifies the anisotropy of light scattering in the superficial layer of a tissue
}

Steven L. Jacques

Stéphane Roussel

Ravikant Samatham 


\title{
Polarized light imaging specifies the anisotropy of light scattering in the superficial layer of a tissue
}

\author{
Steven L. Jacques, ${ }^{a, \star}$ Stéphane Roussel, ${ }^{b}$ and Ravikant Samatham ${ }^{a}$ \\ ${ }^{a}$ Oregon Health \& Science University, Biomedical Engineering, 3303 SW Bond Avenue, Portland, Oregon 97239, United States \\ bUniversité Paris Saclay, Polytech Paris-Sud, 300 Rue du Château, 91400 Orsay, France; LPICM, CNRS, Ecole Polytechnique, \\ 91128 Palaiseau, France
}

\begin{abstract}
This report describes how optical images acquired using linearly polarized light can specify the anisotropy of scattering $(g)$ and the ratio of reduced scattering $\left[\mu_{\mathrm{s}}^{\prime}=\mu_{\mathrm{s}}(1-g)\right]$ to absorption $\left(\mu_{\mathrm{a}}\right)$, i.e., $N^{\prime}=\mu_{\mathrm{s}}^{\prime} / \mu_{\mathrm{a}}$. A camera acquired copolarized $(\mathrm{HH})$ and crosspolarized $(\mathrm{HV})$ reflectance images of a tissue (skin), which yielded images based on the intensity $(I=\mathrm{HH}+\mathrm{HV})$ and difference $(Q=\mathrm{HH}-\mathrm{HV})$ of reflectance images. Monte Carlo simulations generated an analysis grid (or lookup table), which mapped $Q$ and $I$ into a grid of $g$ versus $N^{\prime}$, i.e., $g(Q, I)$ and $N^{\prime}(Q, I)$. The anisotropy $g$ is interesting because it is sensitive to the submicrometer structure of biological tissues. Hence, polarized light imaging can monitor shifts in the submicrometer (50 to $1000 \mathrm{~nm}$ ) structure of tissues. The $Q$ values for forearm skin on two subjects (one Caucasian, one pigmented) were in the range of $0.046 \pm 0.007$ (24), which is the mean \pm SD for 24 measurements on 8 skin sites $\times 3$ visible wavelengths, 470,524 , and $625 \mathrm{~nm}$, which indicated $g$ values of $0.67 \pm 0.07(24)$. () The Authors. Published by SPIE under a Creative Commons Attribution 3.0 Unported License. Distribution or reproduction of this work in whole or in part requires full attribution of the original publication, including its DOI. [DOI: 10.1117/1.JBO.21.7.071115]
\end{abstract}

Keywords: polarized light; anisotropy; scattering; biological tissues; biomedical optics.

Paper 150656SSRR received Oct. 1, 2015; accepted for publication Apr. 18, 2016; published online May 10, 2016.

\section{Introduction}

Polarized light has a long history of providing optical contrast for imaging, which enables views of materials that the eye does not normally see (Refs. 1-7). This report describes how optical images acquired using linearly polarized light can specify the anisotropy of scattering $(g)$ and the ratio of reduced scattering $\left[\mu_{\mathrm{s}}^{\prime}=\mu_{\mathrm{s}}(1-g)\right]$ to absorption $\left(\mu_{\mathrm{a}}\right)$, i.e., $N^{\prime}=\mu_{\mathrm{s}}^{\prime} / \mu_{\mathrm{a}}$. The $g$ is especially interesting because it is sensitive to the submicrometer structure of biological tissues. The size distribution in the 100 to $1000 \mathrm{~nm}$ range affects the angular and wavelength dependence of scattering. Alternatively, the periodicity of mass density, which yields a periodicity in refractive index, strongly scatters light at a wavelength related to the periodicity and the angle between the axis of periodicity and the direction of observation. Tissues present a size distribution of "particle sizes" that scatter light, or a distribution of "periodicities" that scatter light.

Figure 1 shows how $g$ varies versus the size of spherical particles in a medium or tissue, based on Mie theory. Although tissues certainly do not consist of spherical scatterers of a single size, the $g$ value serves as a heuristic descriptor to characterize the tissue as behaving as a medium with a single particle size or a distribution of sizes. Hence, subtle shifts in particle size distribution can be detected. The value of $g$ is related to the dimensionless ratio $d /\left(\lambda / n_{\text {med }}\right)$, where $d$ is the sphere diameter, $\lambda / n_{\text {med }}$ is the wavelength of light in the medium that surrounds the particle, and $n_{\text {med }}$ is the refractive index of the medium. This paper uses $n_{\text {sphere }}=1.38$ and $n_{\text {med }}=1.35$. The $g$ is sensitive to the range of $d /\left(\lambda / n_{\text {med }}\right)=0.1$ to 1 , which corresponds to

*Address all correspondence to: Steven L. Jacques, E-mail: jacquess@ohsu .edu
36 to $365 \mathrm{~nm}$ if using blue-green 500-nm wavelength light in a tissue. However, the scattering from structures in the range $1<d /\left(\lambda / n_{\text {med }}\right)<10$ (e.g., $370 \mathrm{~nm}>d>3.70 \mu \mathrm{m}$ like mitochondria, for 500-nm light), which yields fluctuations in equivalent $g$ values, may be a source of confusion in interpreting a measured $Q$ value in terms of a specific $g$ value (see Sec. 4). Gurjari et al., however, took advantage of such fluctuations to detect the size of nuclei in tissue samples. ${ }^{8}$

Polarized light is randomized by (1) multiple scattering and (2) passage through many local regions of birefringence where each region's fast axis of birefringence is oriented differently. ${ }^{9}$ Hence, deeply penetrating photons have a randomized polarization. Detecting backscattered light that is still polarized selects a superficial layer of tissue for imaging and detection. ${ }^{10,11}$ Ramella-Roman showed that the thickness of this layer depends more on the birefringence of the tissue than on the wavelength of light. ${ }^{12}$ The depolarization coefficient for linear polarization, $\mu_{Q}$ $\left(\mathrm{cm}^{-1}\right)$, scales as approximately $1: 20: 100$ for liver:muscle:skin, respectively. ${ }^{10}$ Hence, tissues present a wide dynamic range of $\mu_{Q}$ values.

An intensity image $(I)$ detected as escaping flux or diffuse reflectance $\left(R_{\mathrm{d}}\right)$ is related to the ratio $N^{\prime}=\mu_{\mathrm{s}}^{\prime} / \mu_{\mathrm{a}}$, which is approximated by diffusion theory, ${ }^{13}$ or by the following expression:

$I=R_{\mathrm{d}}=a_{1} e^{-a_{2} / \sqrt{3\left(N^{\prime}+1\right)}}+a_{3}$,

where $a_{1}=1.0386, a_{2}=6.1206$, and $a_{3}=-0.0488$ for $n_{r}=$ $n_{\text {tissue }} / n_{\text {outside.medium }}=1.38 / 1.0$ for a tissue/air surface boundary, ignoring the effect of a thin glass coverslip that only slightly broadens the light distribution within the tissues. Figure 2 plots Monte Carlo (MC) simulations (stok1.c, ${ }^{14}$ with a tissue/air surface added to allow total internal reflectance) 


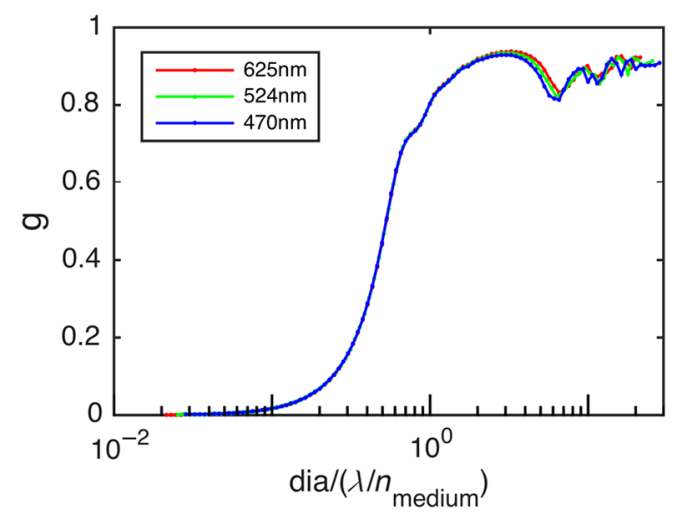

Fig. 1 The anisotropy of scattering $(g)$ is dependent on the diameter (dia) of a spherical particle (mimicking a nanoscale structure within a tissue) and the wavelength $(\lambda)$ of light in the medium (refractive index $n_{\text {medium }}$ ).

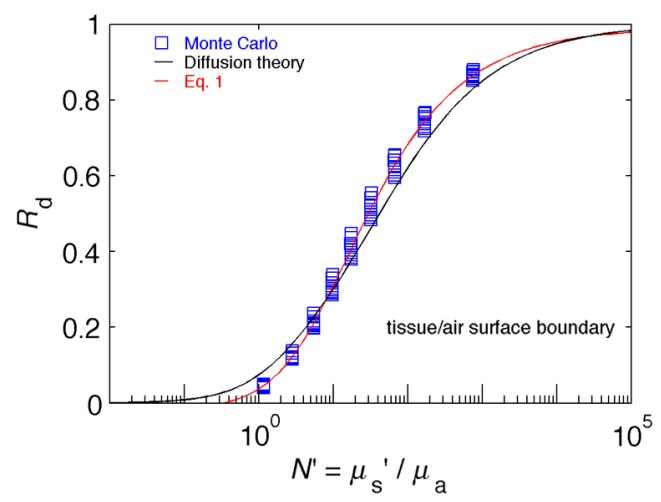

Fig. 2 The blue squares are MC simulations results for diffuse reflectance $\left(R_{\mathrm{d}}\right)$ versus the ratio of reduced scattering to absorption $\left(N^{\prime}=\mu_{\mathrm{s}}^{\prime} / \mu_{\mathrm{a}}\right)$. The black line is diffusion theory. ${ }^{13}$ The red line is Eq. (1). Refractive mismatch at tissue/air surface is $1.38 / 1.00$.

of $R_{\mathrm{d}}$ versus $N^{\prime}$ using a range of $\mu_{\mathrm{a}}$ and $\mu_{\mathrm{s}}^{\prime}$ values and compares with diffusion theory and Eq. (1). The $R_{\mathrm{d}}$ versus $N^{\prime}$ curve applies to any homogeneous semi-infinite medium, whether a phantom or a tissue. For skin with its multiple layers, the curve is approximate, dominated by the dermal optical properties and modified by the melanin absorption in a pigmented epidermis.

This report tests the ability of a pair of $I$ and $Q$ values to specify a $g$ value, which characterizes the submicrometer structure of the tissue. The $I$ value directly specifies the $N^{\prime}$ parameter, so $N^{\prime}$ is no more interesting than the $I$ value itself, but it affects the interpretation of $Q$ to specify $g$. Therefore, $N^{\prime}$ is not emphasized in this paper but is necessary. The paper illustrates the method by applying the analysis to images of ventral and dorsal forearm skin sites using red, green, and blue light-emitting diodes (LEDs) for illumination.

\section{Methods}

\subsection{Polarized Light Camera}

Figure 3 shows the schematic of the experimental setup. The sample was illuminated by LEDs obliquely at $30 \mathrm{deg}$ off normal to the skin surface, which mitigated the amount of specular reflectance reaching the camera that viewed the skin from above. The skin

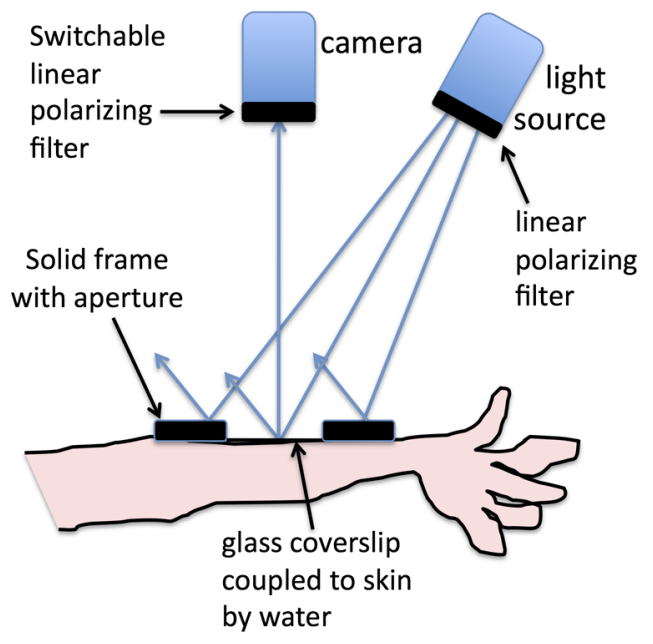

Fig. 3 Polarized light imaging system (schematic drawing). White light was delivered at 30 deg onto a skin site pressed against a solid frame with an aperture. The skin was coupled by water to a glass cover slip spanning the aperture. The camera viewed the skin within the aperture from above.

was coupled by a thin film of water to a glass coverslip, such that specular reflectance from the glass/air surface was directed away from the camera. The light sources were LEDs at center wavelengths of $625 \mathrm{~nm}$ (red), $524 \mathrm{~nm}$ (green), and $470 \mathrm{~nm}$ (blue), with a spectral full-width half-max of $\sim 15 \mathrm{~nm}$, delivering light through a linear polarizer oriented parallel $(\mathrm{H})$ to the scattering plane defined by the source/skin/camera triangle. The camera was a monochrome CCD camera (model: Flea, Point Grey Research Inc., Richmond, British Columbia, Canada), viewing the skin site through a second linear polarizer, whose polarization orientation was manually switchable to either parallel (copolarized, $\mathrm{HH}$ ) or perpendicular (cross-polarized, HV) to the scattering plane. $\mathrm{H}$ indicates polarization parallel to the scattering plane and $\mathrm{V}$ indicates perpendicular to the scattering plane. Two images were acquired, $\mathrm{HH}$ and $\mathrm{HV}$.

Calibration used a series of reflectance standards (Spectralon $^{\mathrm{TM}}$, LabSphere Inc., $R_{\text {std }}=0.02,0.50,0.75,0.99$ ), which demonstrated linearity of detection over the range of reflectances. The mean value of reflected intensity $\left(I_{\text {std }}\right)$ from a Spectralon standard $\left(R_{\text {std }}\right)$ within a central field of view within the aperture served to calibrate the wavelength dependence of the (light source) $\times($ detector responsivity) product. An image of a polyurethane plus titanium dioxide phantom $\left(I_{\mathrm{pu}}\right)$, which was very uniform, provided a correction for any nonuniformity in the illumination. An image with the camera blocked yielded the dark current image $\left(I_{\mathrm{dc}}\right)$. These four images (sample, Spectralon ${ }^{\mathrm{TM}}$, polyurethane, and dark current) were used to yield calibrated images in the dimensionless units of diffuse reflectance [W.detected/W.delivered], referred to as intensity $(I)$, the first element of the Stokes vector $[I Q U V]^{T}$ :

$I=\frac{I_{\text {sample }} \frac{I_{\mathrm{pu}}}{\max \left(I_{\mathrm{pu}}\right)}-I_{\mathrm{dc}}}{\left\langle I_{\mathrm{std}} \frac{I_{\mathrm{pu}}}{\max \left(I_{\mathrm{pu}}\right)}-I_{\mathrm{dc}}\right\rangle} R_{\mathrm{std}}$,

where $\max \left(I_{\mathrm{pu}}\right)$ is the maximum pixel value of the image of the polyurethane phantom image.

There are two types of photons escaping the tissue to be viewed by the camera: (1) deeply penetrating multiply scattered 
photons, whose orientation of linear polarization is randomized, and (2) superficially scattered photons, which undergo only one or two (or few) scatterings and retain much of their original linear polarization. The sum of these two images is called an intensity $(I)$ image, equivalent to a standard diffuse reflectance image $\left(R_{\mathrm{d}}\right)$, and the difference of these two images is called a polarized $(Q)$ image:

$I=\mathrm{HH}+\mathrm{HV}$

$Q=\mathrm{HH}-\mathrm{HV}$.

The $Q$ image can be expressed in another manner:

$\mathrm{HH}=1 / 2$ deep + superficial,

$\mathrm{HV}=1 / 2$ deep,

$Q=\mathrm{HH}-\mathrm{HV}=$ superficial,

which indicates how the multiply scattered deeply penetrating light is canceled in the $Q$ image. Typically, the deeply scattered light constitutes $>90 \%$ of the escaping photons, while the superficially scattered still polarized light constitutes $<10 \%$ of the escaping photons. Half of the deeply scattered light is seen in the $\mathrm{HH}$ image, and the other half is seen in the HV image. The difference image $Q$ cancels this common background light, creating an image using only $Q$ light, which reveals the structure of the superficial tissue layer.

When a sample was imaged, the $Q$ and $I$ pixels were converted to a two-dimensional (2-D) histogram (or heat map) of $Q$ versus $I$, expressed as $n(Q, I)$, where $n$ is the number of pixels within each $Q, I$ bin. The elements of this 2-D histogram were used to calculate the mean values of $Q$ and $I$. This protocol was used for both skin measurements and polystyrene microsphere solution measurements.

\subsection{Polarized Monte Carlo}

A polarized MC simulation was used to study how $I$ and $Q$ reflectance would change as the optical properties of the tissue changed. The analysis used Mie theory for spheres. The optical properties of $\mu_{\mathrm{s}}^{\prime}, \mu_{\mathrm{a}}$, and $g$ were systematically varied to achieve a set of desired $g$ and $N^{\prime}$ values. The optical properties were specified by a series of three steps: (1) choose the diameter of a spherical Mie scattering particle (refractive indices $n_{\text {particle }}=1.38$, $n_{\text {medium }}=1.35$ ) to achieve a desired $g$, (2) choose an absorption $\mu_{\mathrm{a}}$ (both $\mu_{\mathrm{a}}=0.1$ and $1 \mathrm{~cm}^{-1}$ were tested and were consistent, but only data from $\mu_{\mathrm{a}}=1 \mathrm{~cm}^{-1}$ are shown), and (3) choose a volume fraction of spheres $\left(f_{\mathrm{v}}\right)$ that corresponds to a concentration or number density of scatterers $\left[\rho_{\mathrm{s}}=f_{\mathrm{v}} / V_{\mathrm{sph}}\left(\# / \mu \mathrm{m}^{3}\right)\right.$, where $V_{\text {sph }}=$ volume of one sphere] that yields a scattering coefficient, $\mu_{\mathrm{s}}=\rho_{\mathrm{s}} \sigma_{\mathrm{s}}\left[\sigma_{\mathrm{s}}\right.$ is the scattering cross-sectional area of a sphere $\left.\left(\mu \mathrm{m}^{2}\right)\right]$, which yields a reduced scattering coefficient, $\mu_{\mathrm{s}}^{\prime}=\mu_{\mathrm{s}}(1-g)\left(10^{4} \mu \mathrm{m} / \mathrm{cm}\right)\left[\mathrm{cm}^{-1}\right]$, such that the desired value of $N^{\prime}=\mu_{\mathrm{s}}^{\prime} / \mu_{\mathrm{a}}$ is obtained. The MC program was the "meridian" method of Ramella-Roman et al., ${ }^{14}$ with a mismatched air/ tissue boundary added, which propagates a Stokes vector $\mathrm{S}=[I Q U V]^{T}$ :

$$
S=\left|\begin{array}{c}
I \\
Q \\
U \\
V
\end{array}\right|=\left|\begin{array}{c}
H+V \\
H-V \\
P_{+45^{\circ}-P_{-45^{\circ}}}^{R-L}
\end{array}\right| .
$$

The program repeatedly launches a photon bundle of $\mathrm{H}$ light as a Stokes vector $\mathrm{S}$, for which $\mathrm{S}=\left[\begin{array}{llll}1 & 1 & 0 & 0\end{array}\right]^{T}$ (superscript $T$ indicates the vector is transposed into a vertical column vector). As light escapes at the front surface of the tissue, the escaping weight of the photon bundle scales the elements $I, Q, U$, and $V$ of the Stokes vector escaping the tissue, which is recorded. After a number of photons $\left(N_{\text {photons }}\right)$ have been launched, the total escaped intensities of the $I, Q, U$, and $V$ values are divided by $N_{\text {photons. }}$ Hence, the results are expressed as a fraction of the total incident light ( $W$ observed per $W$ delivered) or (dimensionless) that escapes as reflectance. This report used only the $I$ and $Q$ outputs.

Figure 4(a) shows the output of data from the MC simulation portrayed as a 3-D plot of observable $I$ and $Q$ for a range of $g$ values. Figure 4(b) shows the view of the same data as a $2-D$ plot of $g$ versus $I$. The curves are vertical because the number density $\rho_{\mathrm{s}}$ was chosen to achieve one of a set of nine $I$ values from 0.1 by 0.1 to 0.9 . The curves are not perfectly vertical, likely because diffusion theory was used to choose $\rho_{\mathrm{s}}$. Figure 4(c) shows the view of $Q$ versus $I$. The black lines are iso-g contours drawn using Eqs. (3) and (4):

$Q=a_{1}\left[1-\exp \left(-a_{2} I\right)\right]$

where $a_{1}(g)$ and $a_{2}(g)$ were fit by least-squares regression of MC data. A subroutine $\operatorname{getQ}(I, g)$ that implements Eq. (9) is listed in the Appendix. Figure 4(d) shows $Q$ versus $g$ for the range of $I$ values. The fluctuation in $Q$ for $g>0.7$ is apparent.

\subsection{Polarized Light Camera Images on Microsphere Phantoms}

To verify the accuracy of the MC simulations, experiments on a set of polystyrene sphere solutions were conducted (sphere diameters of 100, 170, 200, 260, 300,360, 430, and $770 \mathrm{~nm}$, prepared in water with sonication to avoid sphere aggregation). Solutions were placed in six-well petri dishes $(3 \mathrm{~cm}$ wide by $1.5 \mathrm{~cm}$ deep) and imaged with the red, green, and blue LEDs. The protocol for obtaining the average $Q$ of the solutions was the same as for skin sites. Figure 5 shows the experimental values of $Q$ versus $g$ as colored circles.

MC simulations were also conducted for the three LED wavelengths using the refractive index of polystyrene (1.582, 1.589 , and 1.596) and water $(1.339,1.337$, and 1.333) at the center wavelengths of the three LEDs (center wavelengths of $470,524,625 \mathrm{~nm}$; full-width half-max $\approx 15 \mathrm{~nm}$ ) and a series of sphere sizes and number densities. In Fig. 5, the three black lines are the predictions of the MC simulations for the three LED wavelengths, which superimpose, indicating that $Q(g)$ is not dependent on wavelength. The theoretical curves match the experimental data.

\subsection{Polarized Light Camera Images on Skin}

Ventral and dorsal forearm skin sites on the left and right forearms were imaged on two subjects, one Caucasian (Fitzpatrick skin type I) and one pigmented (skin type III), for a total of eight sites. The skin was coupled to the overlying glass coverslip by a 


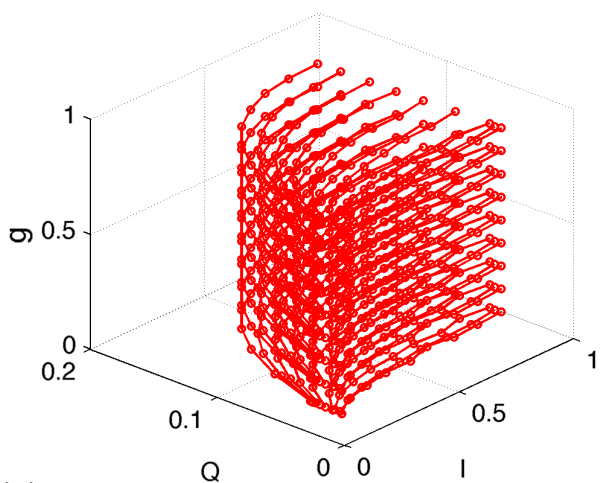

(a)

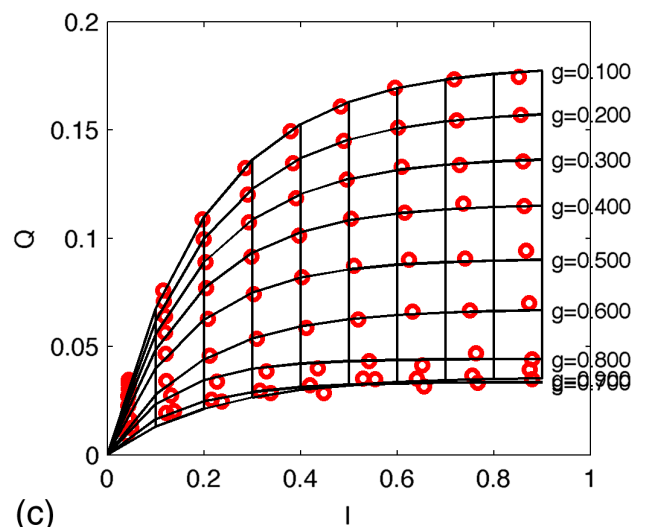

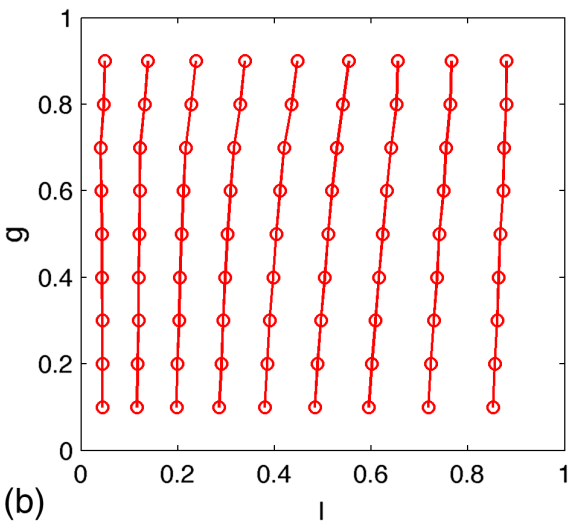

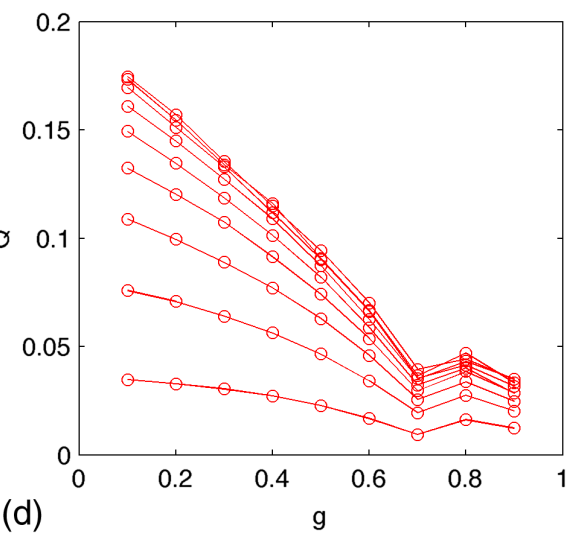

Fig. 4 The $I, Q$, and $g$ values for MC simulations, where $g$ is specified by sphere diameter (dia) using Mie theory, and sphere density, $\rho_{\mathrm{s}}\left[\# / \mu \mathrm{m}^{3}\right]$, is adjusted to achieve a target value of reflected intensity $(I)$. (a) 3-D plot. Data are for $\mu_{\mathrm{a}}=1 \mathrm{~cm}^{-1}$. Data for $\mu_{\mathrm{a}}=0.1 \mathrm{~cm}^{-1}$ (not shown) were consistent with the results for $\mu_{\mathrm{a}}=1 \mathrm{~cm}^{-1}$. (b) $g$ versus $I$. The adjustment of the $\rho_{\mathrm{s}}$ to yield desired $I$ values was based on diffusion theory, which slightly deviates from polarized MC simulations, so the curves are not perfectly vertical. (c) $Q$ versus $I$. The black lines are iso- $g$ curves generated by the subroutine getQ $(I, g)$ (see Appendix). (d) $g$ versus $Q$. Each curve is a different $I$ (higher $I$ yields higher $Q$ ). There is a fluctuation in $Q$ at $g>0.7$, which may complicate the determination of $g$ by very low $Q$ values.

film of water. The skin/water/glass/air interface avoided specular reflectance from the skin/air interface, and the oblique illumination caused the much lower specular reflectance from the skin/water interface to be directed away from the camera. Since the change in an escaping photon's trajectory angle at the skin/ glass interface was undone by the change in angle at the glass/air

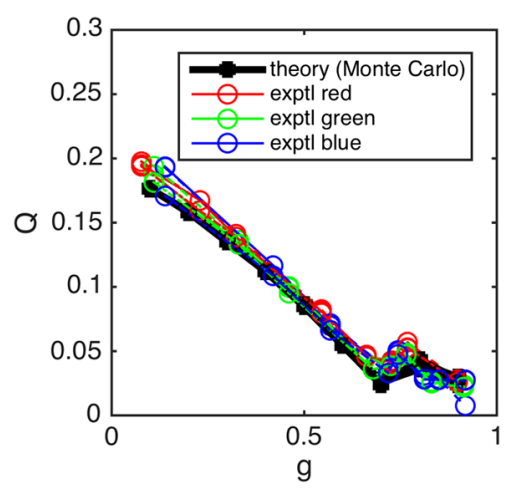

Fig. $5 Q$ versus $g$ for polystyrene microspheres of various size in water at each of the three LED wavelengths. Three black lines show MC results for the spheres at the three LED wavelengths, which superimpose. interface, the refractive index of the glass did not affect total internal reflectance, which was governed by the skin/air mismatch. Images were acquired with red, green, and blue LED illumination, for a total of 24 sets of $I$ and $Q$ images.

\section{Results}

The mean values of $Q$ and $I$ for each skin site are summarized in Fig. 6. Figure 6(a) shows a bar graph of the mean $Q$ and $I$ values, which illustrates how the pigmentation in the pigmented subject attenuated $I$ but had little effect on $Q$. The $Q$ data were consistently lower for blue than for red light (see Sec. 4). Figure 6(a) also shows that the pigmented subject attenuated the total reflected intensity $(I)$ more than the Caucasian subject, but pigmentation had less effect on the polarized intensity $Q$. Figure 6(b) plots the mean $Q$ values versus the mean $I$ values. The grid (black lines) used the analysis grid of Fig. 4(c). Table 1 summarizes the mean $Q$ and $I$ values, and the corresponding $g$ values. The $Q, I$ data indicate that $g$ is distributed around $0.67 \pm 0.07$ (mean $\pm \mathrm{SD}, n=24$ measurements on all sites using all LEDs).

\section{Discussion}

The importance of specifying the anisotropy $g$ is its sensitivity to the submicrometer structure of cells and tissues. For example, the remodeling of collagen gels by matrix metalloproteinases 
(a)
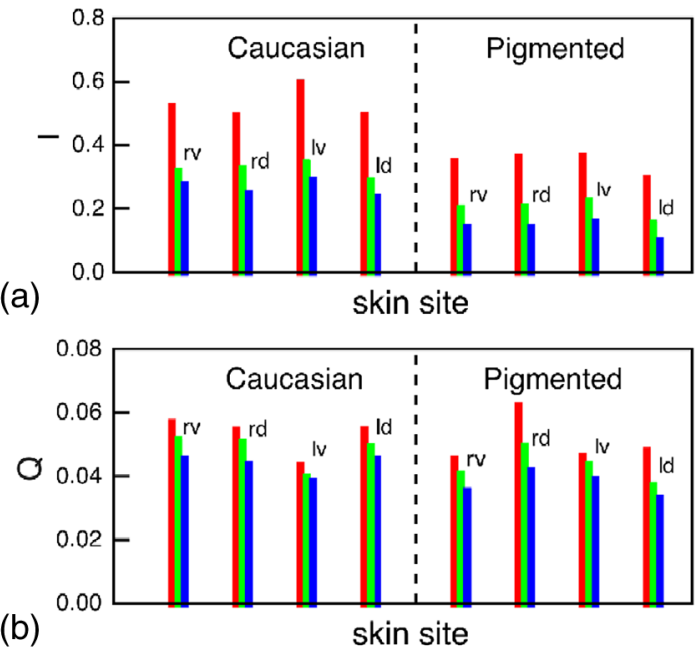

(b)

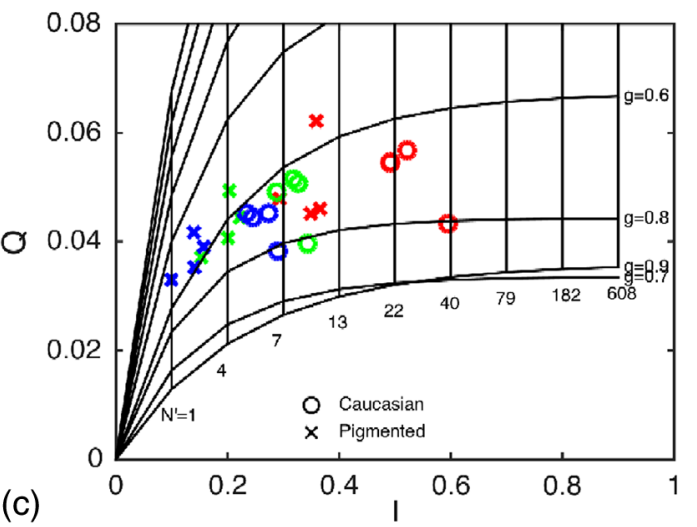

Fig. 6 Skin measurements. (a) The mean values of $Q$ for eight skin sites [ventral, dorsal, left, and right forearm on two individuals, Caucasian (o) and pigmented (x)], using red, green, and blue LEDs (indicated by symbol color). (b) The mean values of $l$. (c) Plot of $Q$ versus / for the skin sites. The grid (black lines) of $g$ versus $N^{\prime}$ used the function $Q=\operatorname{get} Q(I, g)$, which is based on polarized $\mathrm{MC}$ simulations for spheres with refractive index 1.38 in medium with refractive index 1.35 .

from cultured cells causes $g$ to drop as fiber bundles are broken down to small fibrils. ${ }^{15,16}$ The effect of a gene mutation, osteogenesis imperfecta, in mouse skin is to drop $\mathrm{g}$, as collagen fibrils fail to aggregate properly into fiber bundles. ${ }^{17}$ Optical clearing of dermis using glycerol causes a drop in $g,{ }^{18}$ which is tentatively thought to be due to increased fibril packing and order as the glycerol dessicates the collagen fiber bundles. A more ordered fibril packing can allow constructive interference of scattered wavelengths from the fibrils, analogous to how the cornea of a fish eye is clear but upon death becomes cloudy as it loses its active control of hydration and becomes disordered. The above example studies used confocal reflectance microscopy to specify $g$. Polarized light imaging may be a more rapid and convenient method for assessing submicrometer changes in the structure of in vivo tissue sites over large fields of view. For example, the remodeling of collagen in aging skin could perhaps be assayed by such a noninvasive $g$ measurement using polarized light.

The $Q$ for blue light was lower than the $Q$ for red light. One possible explanation is that the scattering coefficient is higher for blue light than for red light, so there were more photon/tissue interactions, allowing more depolarization. Another possible
Table 1 Mean I and $Q$ values for skin sites. $r, I=$ right, left forearm; $\mathrm{v}, \mathrm{d}=$ ventral, dorsal forearm. Illumination used red $(625 \mathrm{~nm})$, green (524 $\mathrm{nm})$, and blue (470 $\mathrm{nm})$ LEDs.

\begin{tabular}{|c|c|c|c|c|}
\hline Skin site & LED & I & $Q$ & $g$ \\
\hline Caucasian rv & Red & 0.522 & 0.057 & 0.664 \\
\hline Caucasian rv & Green & 0.318 & 0.052 & 0.646 \\
\hline Caucasian rv & Blue & 0.273 & 0.045 & 0.699 \\
\hline Caucasian rd & Red & 0.492 & 0.054 & 0.683 \\
\hline Caucasian rd & Green & 0.326 & 0.051 & 0.663 \\
\hline Caucasian rd & Blue & 0.246 & 0.044 & 0.680 \\
\hline Caucasian Id & Red & 0.492 & 0.055 & 0.682 \\
\hline Caucasian Id & Green & 0.288 & 0.049 & 0.654 \\
\hline Caucasian Id & Blue & 0.234 & 0.045 & 0.649 \\
\hline Caucasian Iv & Red & 0.595 & 0.043 & 0.804 \\
\hline Caucasian Iv & Green & 0.343 & 0.040 & 0.787 \\
\hline Caucasian Iv & Blue & 0.290 & 0.038 & 0.790 \\
\hline Pigmented rv & Red & 0.349 & 0.045 & 0.749 \\
\hline Pigmented rv & Green & 0.201 & 0.041 & 0.674 \\
\hline Pigmented rv & Blue & 0.141 & 0.035 & 0.610 \\
\hline Pigmented rd & Red & 0.359 & 0.062 & 0.578 \\
\hline Pigmented rd & Green & 0.203 & 0.049 & 0.574 \\
\hline Pigmented rd & Blue & 0.140 & 0.042 & 0.559 \\
\hline Pigmented Id & Red & 0.293 & 0.048 & 0.678 \\
\hline Pigmented Id & Green & 0.153 & 0.037 & 0.609 \\
\hline Pigmented Id & Blue & 0.100 & 0.033 & 0.556 \\
\hline Pigmented IV & Red & 0.366 & 0.046 & 0.744 \\
\hline Pigmented IV & Green & 0.222 & 0.044 & 0.643 \\
\hline Pigmented IV & Blue & 0.157 & 0.039 & 0.594 \\
\hline Mean values & & 0.296 & 0.046 & 0.665 \\
\hline Standard deviations & 0.130 & 0.0071 & 0.071 & \\
\hline
\end{tabular}

explanation is that blue light interrogated only the papillary dermis with smaller collagen fiber bundles, while red light also interrogated the upper reticular dermis with larger collagen fiber bundles. Smaller structures with lower anisotropy of scattering depolarize more efficiently than larger structures with higher anisotropy of scattering.

Two limitations of the polarized light method presented in this paper deserve mention. First, the analysis assumes that the tissue is homogenous in its optical properties. For some tissues, this is not a bad approximation, but in other tissues, there are definite tissue layers with different optical properties. The 
next task in this project is to implement a two-layer model, in which a superficial layer of specified thickness and optical properties sits on top of an underlying layer of different optical properties. The current homogeneous-tissue MC model for polarized light propagation is being updated to a heterogeneous model, which will allow characterization of superficial lesions that sit on top of an underlying normal dermis.

The second limitation is that the method may become confusing at high values of $g$ exceeding $\sim 0.65$, due to the fluctuations in $Q$ versus $g$. Does an observed $Q$ of 0.047 indicate $g=0.67$ or $g=0.80$ ? Skin showed values of $g$ in the range of 0.60 to 0.70 , and since the distribution of $Q$ extended down to 0.60 , it is probable that the skin is due to $g \approx 0.67$, not 0.80 . This paper's analysis was based on Mie theory using single scatterer sizes. Mixtures of scatterer sizes may not yield oscillatory behavior of $g$, and future work will explore the calibration of $g(Q, I)$ using mixtures of spheres.

In summary, images of $\mathrm{HH}$ and $\mathrm{HV}$ yield images of $Q$ and $I$, which can specify an apparent anisotropy $g$ that serves as a metric to characterize a tissue as behaving equivalently to a solution of Mie scatterers of one size, for descriptive purposes only. More work is needed to understand the interpretation of measured $Q$ and $I$ in terms of $g$. Nevertheless, $Q$ will be sensitive to changes in tissue ultrastructure and should be a useful noninvasive imaging modality for evaluating in vivo tissue sites.

\section{Appendix}

The following is a MATLAB ${ }^{\text {TM }}$ subroutine that yields the value of $Q$ using the arguments of diffuse reflected intensity $(I)$ and anisotropy of scatter $(g)$. The subroutine is based on fitting the $Q$ versus $I \mathrm{MC}$ data for spheres with $n_{\text {sphere }}=1.38$ and $n_{\text {medium }}=1.35$, and a tissue/air interface, $n_{\text {medium }} / n_{\text {air }}=$ $1.35 / 1.00$, for each choice of $g$ by Eq. (9), to yield parameters $a_{1}$ and $a_{2}$.

function $Q=\operatorname{get} Q(I, g)$

$\mathrm{aa}=[\% \mathrm{a} 1, \mathrm{a} 2$

$0.1800 \quad 4.7067 \quad \% \mathrm{~g}=0.1$

$0.1590 \quad 4.9364 \% \mathrm{~g}=0.2$

$0.13765 .1901 \% \mathrm{~g}=0.3$

$0.11595 .4310 \% \mathrm{~g}=0.4$

$0.09055 .8528 \% g=0.5$

$0.06735 .3231 \% g=0.6$

$0.0336 \quad 6.6901 \% \mathrm{~g}=0.7$

$0.04437 .5464 \% \mathrm{~g}=0.8$

$0.0360 \quad 4.4602 \% \mathrm{~g}=0.9$

] ;

a1 $=$ aa $(:, 1) ;$ o vector of values

$a 2=a a(:, 2) ; \%$ a vector of values

$g g=[.1: .1: .9]^{\prime} ;$ \% a vector of values for anisotropy

$\mathrm{QQ}=\mathrm{a} 1 . *(1-\exp (-I \times \mathrm{a} 2)) ; \%$ a vector of values for $Q$

\%. * implies element by element multiplication of two vectors

$Q=$ interpl $(\mathrm{gg}, \mathrm{QQ}, \mathrm{g}) ; \%$ linear interpolation $Q$ versus gg for specific $g$

\section{References}

1. M. J. Raković et al., "Light backscattering polarization patterns from turbid media: theory and experiment," Appl. Opt. 38(15), 3399-3408 (1999).

2. J. R. Mourant, T. M. Johnson, and J. P. Freyer, "Characterizing mammalian cells and cell phantoms by polarized backscattering fiber-optic measurements," Appl. Opt. 40(28), 5114-5123 (2001).

3. V. Sankaran, J. T. Walsh, Jr., and D. J. Maitland, "Comparative study of polarized light propagation in biologic tissues," J. Biomed. Opt. 7(3), 300-306 (2002).

4. M. Todorović et al., "In vivo burn imaging using Mueller optical coherence tomography," Opt. Express. 16(14), 10279-10284 (2008).

5. M. R. Antonelli et al., "Mueller matrix imaging of human colon tissue for cancer diagnostics: how Monte Carlo modeling can help in the interpretation of experimental data," Opt. Express 18(10), 10200-10208 (2010).

6. S. Alali and A. Vitkin, "Polarized light imaging in biomedicine: emerging Mueller matrix methodologies for bulk tissue assessment," $J$. Biomed. Opt. 20(6), 061104 (2015).

7. C. M. Macdonald, S. L. Jacques, and I. V. Meglinski, "Circular polarization memory in polydisperse scattering media," Phys. Rev. E Stat. Nonlinear Soft Matter. Phys. 91(3), 033204 (2015).

8. R. S. Gurjari et al., "Imaging human epithelial properties with polarized light- scattering spectroscopy," Nat. Med. 7(11), 1245-1248 (2001).

9. S. L. Jacques, "Polarized light imaging of biological tissues," Chapter 30 in CRC Handbook of Biomedical Optics, D. A. Boas, C. Pitris, and N. Ramanujam, Eds., pp. 649-669, CRC Press, Taylor \& Francis Group, Boca Raton, Florida (2011).

10. S. L. Jacques, J. Roman, and K. Lee, "Imaging superficial tissues with polarized light," Lasers Surg. Med. 26, 119-129 (2000).

11. S. L. Jacques, J. C. Ramella-Roman, and K. Lee, "Imaging skin pathology with polarized light," J. Biomed Opt. 7, 329-340 (2002).

12. J. C. Ramella-Roman, "Imaging skin pathologies with polarized light: empirical and theoretical studies," PhD Dissertation, Oregon Health \& Science University (2004).

13. T. J. Farrell, M. S. Patterson, and B. Wilson, "A diffusion theory model of spatially resolved, steady-state diffuse reflectance for the noninvasive determination of tissue optical properties in vivo," Med. Phys. 19(4), 879-888 (1992)

14. J. C. Ramella-Roman, S. A. Prahl, and S. L. Jacques, "Three Monte Carlo programs of polarized light transport into scattering media: part I," Opt. Express 13(12), 4420-4438 (2005).

15. D. Levitz et al., "Quantitative characterization of developing collagen gels using optical coherence tomography," J. Biomed. Opt. 15(2), 026019 (2010).

16. D. Levitz et al., "Non-destructive label-free monitoring of local smooth muscle cell remodeling of collagen gels using optical coherence tomography," Biomaterials 31(32), 8210-8217 (2010).

17. R. Samatham, S. L. Jacques, and P. Campagnola, "Optical properties of mutant vs wildtype mouse skin measured by reflectance-mode confocal scanning laser microscopy (rCSLM)," J. Biomed. Opt. 13(4), 041309 (2008).

18. R Samatham, K. G. Phillips, and S. L. Jacques, "Assessment of optical clearing agents using reflectance-mode confocal scanning laser microscopy,' J. Innovative Opt. Health Sci. 3(3), 183-188 (2010).

Steven L. Jacques is a professor of biomedical engineering at the Oregon Health \& Science University (OHSU). He received his BS degree in biology from MIT in 1972 and his MSEE and PhD degrees from the University of California-Berkeley in 1975 and 1983. He is a SPIE fellow.

Stéphane Roussel is a student at the Polytech Paris-Sud and Ecole Polytechnique, France, who spent a summer internship at OHSU.

Ravikant Samatham is a research associate at the Oregon Health \& Science University. He received his BTech at JNTU College of Engineering, Anantapur, India. He received his MS at the University of Nevada-Reno and his PhD at the Oregon Health \& Science University. He built the polCAM. 